\title{
Using Gudermannian to Improve the Turbo-Code Mathematical Principles in 3G Communication Systems
}

\author{
Yuriy Ivanov, Anatoliy Kulyk, Alexander Romanyuk \\ Institute of Automatics, Electronics and Computer Control Systems, Vinnytsia National Technical \\ University, Khmelnytske shosse, 95, Vinnytsia, 21021, Ukraine, \\ e-mail: YuraII@yandex.ru,kulyk@inaeksu.vntu.edu.ua,ran12345@mail.ru
}

\begin{abstract}
This paper discusses the promising modern turbocodes in the concept of $3^{\text {rd }}$ generation comunication systems. Presents the extended mathematical principles of the algebra logarithm of the likelihood function for $N$ statistically independent information bits with the transition to trigonometric and hyperbolic functions by using Gudermannian expression and decomposition in B. Taylor power series for finding the result with the defined accuracy.
\end{abstract}

Index Terms-Turbo-code, 3G communication systems, LogLikelihood Ratio, Gudermannian, B. Taylor power series.

\section{INTRODUCTION}

T the time of the latest technology information became the object of automated processing. The data transmission process in information systems is susceptible to errors, because any error is the material calculation breach. To combat interferences in data transmission systems at all the stages error-correction coding is used, that provides reliability and credibility of transmitted information. The analysis allowed us to determine, that the most prominent achievement in the theory of error-correction coding in recent years is turbo-code - the powerful algorithm of the modern communication systems [1]. This code is the iterative probabilistic method of error-correcting coding with the reliable performance, which is very close to the Claude Shannon theoretical limit. Turbo-codes are used to encode large volume information messages at the high speed with high error-correcting [1], [2]. These codes are used in practice in the most important areas, such as space satellite communications (standards DVB-S, DVB-RCS), digital television (ViaSat, HDTV), $3^{\text {rd }}$ generation mobile communication systems [3]. The practical widespread have decoding algorithms: SOVA (Soft-Output Viterbi Algorithm), MAP (decoding algorithm for the Maximum A posteriori Probability) and modifications to decrease the computational complexity (Log-MAP, Max-Log-MAP) [4].

The authors are with the Institute of Automatics, Electronics and Computer Control Systems, Vinnytsia National Technical University, Vinnytsia, Ukraine (e-mail: YuraII@yandex.ru,kulyk@inaeksu.vntu.edu.ua).
The main problems are the lack of freeware distribution and the decoding algorithms complexity [2], [4].

The work objective is the calculation efficiency improvement of the turbo-code decoding procedure through the betterment of the mathematical algorithms of their work in 3G communication systems.

The research subject is the mathematical algorithms for decoding turbo-codes in $3 \mathrm{G}$ communication systems.

To achieve the work objective it is necessary to solve the following tasks:

$>$ to explore the work of promising modern turbo-codes in $3 \mathrm{G}$ communication systems;

$>$ to build a model of error-correction turbo-decoder which is uses the extended mathematical principles of the algebra logarithm of the likelihood function for $N$ statistically independent information bits with the transition to trigonometric and hyperbolic functions without using complex numbers.

\section{CONCEPT OF THE $3^{\mathrm{RD}}$ GENERATION SYSTEMS}

The concept of the $3^{\text {rd }}$ generation systems lies in the creating conditions for the multimedia services provision, including the high speed information transmission, such as video, audio, fax messages or other data to any subscriber by using the mobile terminal. The cost should be minimal, and to have the acceptable quality and safety level [5].

The $3^{\text {rd }}$ generation services package: voice communication (Voice-mail), low speed data exchange (Short Messaging Service, Location Based Service), interactive data exchange (Videotelephony, transmission of the large information volume), asymmetric multimedia data transmission (work on the Internet and Intranet) [3], [5].

It is necessary to provide defined bit rates for different subscriber mobility degrees depending on the coverage area size:

$>$ up to $2048 \mathrm{Kbit} / \mathrm{s}$ for low subscriber mobility and the local coverage area;

$>$ up to $144 \mathrm{Kbit} / \mathrm{s}$ for high subscriber mobility (speed up to $120 \mathrm{~km} / \mathrm{h}$ ) and the wide coverage area ; 
up to 64 (144) Kbit/s for the global coverage (satellite communication).

The architecture of such systems includes two main elements: Access Network and Core Network. This structure provides the ability to build infrastructure by the successive components modifications [3].

Turbo-codes are widely used in $3^{\text {rd }}$ generation mobile communication systems. For example, $3 \mathrm{G}$ networks operate on the UHF frequencies, usually in the range about $2 \mathrm{GHz}$, and transmite data with the speed up to 3,6 Mbit/s. They allow to organize Videotelephony, to view on the mobile phone movies and TV shows. $3 \mathrm{G}$ includes 5 standards of IMT-2000 family (UMTS/WCDMA, CDMA2000/IMT-MC, TDCDMA/TD-SCDMA, DECT, UWC-136). Two standards are the most popular in the world: UMTS (W-CDMA) and CDMA2000 (IMT-MC), which are based on one and the same technology - Code Division Multiple Access [6].

The important advantage of the turbo-codes is the decoding complexity independence from the information block length, which reduces the decoding error probability by increasing its length.

The significant drawback of the turbo-codes is comparatively high decoding complexity and high delay, which sometimes make them unattractive. But for using in satellite channels this shortcoming is not essential, because the communication channel length introduces the significant delay. Another drawback of the turbo-codes is the comparatively small code distance. This leads to the fact, that even when a large input error probability, the turbo-code performance is high, but at the low input error probability the turbo-code performance is limited [3], [5], [6].

\section{EXPANSION OF THE ALGEBRA LOGARITHM OF THE LIKELIHOOD FUNCTION}

To simplify calculations the computational procedure is used, that is called Log-Likelihood Ratio (LLR) [4]

$$
\operatorname{LLR}(x)=\ln \left(\frac{p(x=+1)}{p(x=-1)}\right)
$$

where $x$ is received information symbol (statistically independent information bit).

By working with formula (1), by performing substitutions, which are based on the operations with the probabilistic values, by using the mathematical logarithm definition the expression for calculating the Log-Likelihood Ratio for many statistically independent information bits can be written in the form [7]

$$
\begin{aligned}
\sum_{\substack{[+] \\
i=1}}^{N} \operatorname{LLR}\left(x_{i}\right) & =\ln \left(\prod_{i=1}^{N} \exp \left(\operatorname{LLR}\left(x_{i}\right)+1\right)+\right. \\
& \left.+\prod_{i=1}^{N} \exp \left(\operatorname{LLR}\left(x_{i}\right)-1\right)\right)- \\
& -\ln \left(\prod_{i=1}^{N} \exp \left(\operatorname{LLR}\left(x_{i}\right)+1\right)-\right. \\
& \left.-\prod_{i=1}^{N} \exp \left(\operatorname{LLR}\left(x_{i}\right)-1\right)\right),
\end{aligned}
$$

where $[+]$ is sum of the two LLR.

The new model of error-correction turbo-decoder with the new mathematical principles of the algebra logarithm of the likelihood function for $N$ statistically independent information bits has been proposed. This model allows:

$>$ to work with trigonometric (L. Euler, F. Viete, N. Copernicus, Arabic mathematics) and hyperbolic (A. de Moivre, V. Riccati, J. H. Lambert) functions

$$
\sum_{\substack{[+] \\ i=1}}^{N} \operatorname{LLR}\left(x_{i}\right)=2 \cdot \operatorname{arth}\left(\prod_{i=1}^{N} \operatorname{th}\left(\frac{\operatorname{LLR}\left(x_{i}\right)}{2}\right)\right) .
$$

$>$ to work without complex numbers by using C. Gudermann function [8]

$$
g d(\varphi)=\int_{0}^{\varphi} \frac{d t}{c h t}=2 \cdot \operatorname{arctg} \exp (\varphi)-\frac{\pi}{2}
$$

Applying the Gudermannian expression to the formula (3) can be written in the form

$$
\begin{aligned}
& \sum_{\substack{[+] \\
i=1}}^{N} \operatorname{LLR}\left(x_{i}\right)= \\
& \quad=2 \cdot \operatorname{arth}\left(\prod_{i=1}^{N} \operatorname{tg}\left(\operatorname{arctg} \exp \left(\operatorname{LLR}\left(x_{i}\right)\right)-\frac{\pi}{4}\right)\right) .
\end{aligned}
$$

The decomposition in B. Taylor power series for processing in computing devices, finding the result with the defined accuracy will look like this

$$
\begin{aligned}
& \sum_{\substack{[+] \\
i=1}}^{N} \operatorname{LLR}\left(x_{i}\right)= \\
& =2 \cdot \sum_{n=0}^{\infty} \frac{\left(\prod_{i=1}^{N} \operatorname{tg}\left(\operatorname{arctg}\left(\sum_{n=0}^{\infty} \frac{\left(\operatorname{LLR}\left(x_{i}\right)\right)^{n}}{n !}\right)-\frac{\pi}{4}\right)\right)^{2 \cdot n+1}}{2 \cdot n+1},
\end{aligned}
$$


where $-1<\prod_{i=1}^{N} \operatorname{tg}\left(\operatorname{arctg}\left(\sum_{n=0}^{\infty} \frac{\left(L L R\left(x_{i}\right)\right)^{n}}{n !}\right)-\frac{\pi}{4}\right)<1$.

These mathematical principles will help to create the model of error-correction turbo-decoder with high computing efficiency.

\section{CONCLUSION}

The concept of $3 \mathrm{G}$ mobile communication is directed at the creating for the subscribers high speed and quality services. The turbo-codes - powerful algorithms of the modern communication systems - are the best way to perform these conditions. In the cascade scheme, such as turbo-code for reliable work the decoding algorithm should not be limited by the "hard" decisions scheme. Therefore, to make better use of the information obtained from each decoder it is necessary to apply the "soft" decoding scheme based on the mathematical apparatus of the algebra logarithm of the likelihood function. In this paper formulas for the accurate LLR estimation in the case of the few statistically independent information bits for the algebra logarithm of the likelihood function were generalized in $3 \mathrm{G}$ systems to improve computing efficiency of the turbo-code decoding procedure.

\section{REFERENCES}

[1] C. Berrou, A. Glavieux, and P. Thitimajshima, "Near Shannon Limit Error-Correcting Coding and Decoding: Turbo-Codes," in ICC'93, Geneva, Switzerland, May 93, pp. 1064-1070.

[2] Y. Ivanov, A. Kulyk, and S. Krivogubchenko, "A Viterbi Algorithm as a Key to Decoding Turbo-Code," Nauka i studia, vol. 56, no. 11, pp. 60-65, Sep. 2012.

[3] K. Sripimanwat, Turbo Code Applications: A Journey from a Paper to Realization. New York: Springer, 2005, part 4

[4] R. Morelos-Zaragoza, The Art of Error Correcting Coding, 2nd Edition. Chippenham: John Wiley \& Sons Ltd, 2006, ch. 7.
[5] L. Hanzo, L-L. Yang, E-L. Kuan, and K. Yen, Single- and Multi-Carrier CDMA. Multi-User Detection, Space-Time Spreading, Synchronisation and Standards. New York: John Wiley \& Sons Ltd, 2003, part. 5.

[6] L. Hanzo, T. H. Liew, and B. L. Yeap, Turbo Coding, Turbo Equalisation and Space-Time Coding for Transmission over Wireless Channels. Southampton: Department of Electronics and Computer Science of UK, 2002, part. 2.

[7] T. K. Moon, Error Correction Coding: Mathematical Methods and Algorithms. New Jersey: John Wiley \& Sons Ltd, 2005, part 4.

[8] M. Abramowitz and I. A. Stegun, Handbook of Mathematical Functions With Formulas, Graphs, and Mathematical Tables. New York: Dover Publications Inc, 1965, ch. 4.

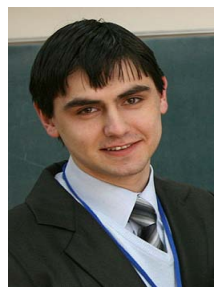

Yuriy Ivanov was born in Ukraine in 1990 . He received the bachelor of engineering degree from Vinnytsia National Technical University, Vinnytsia, Ukraine in 2012.

$\mathrm{He}$ worked with his master of engineering thesis. His research interests include errorcorrection coding, optimization tasks, special mathematics sections.

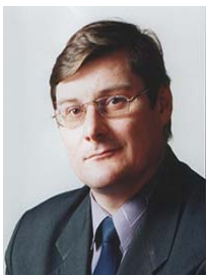

Anatoliy Kulyk was born in USSR in 1960 . He received the engineering degree from the Vinnytsia Technical University, Vinnytsia, Ukraine in 1992.

He joined the Vinnytsia Technical University, Vinnytsia, USSR in 1984, where he is currently professor at the Faculty of Automation and Computer Engineering. He was the engineer at the Institute of Control Problems (Academy of Sciences, Moscow, USSR) in 1984. His research interests include information transmission, errorcorrection and adaptive coding, signal processing.

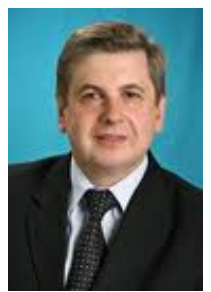

Alexander N. Romanyuk has receieved a $\mathrm{PhD}$ degree in 1986 and Grand $\mathrm{PhD}$ in 2009. At the moment he's author of 350 scientific publications, including 4 monographs and 60 patents. He was appointed on the position of Corresponding member of Telecommunications and Computer Science Academy in Russia.Within recent years he was scientific supervisor of 14 scientific projects. He applied into industry 2 devices - graphics accelerators. He has tight scientific relations with scientists in Russia, Canada, USA, Poland, Romania and Sweeden. 